\title{
Solid damping in micro electro mechanical systems
}

\section{Raffaele Ardito • Claudia Comi . \\ Alberto Corigliano • Attilio Frangi}

Published online: 23 May 2008

(C) Springer Science+Business Media B.V. 2008

\section{Errata-corrige to: Meccanica}

DOI 10.1007/s1012-007-9105-3

\section{ERRATA}

Eq. 2: $\rho C \dot{T}=-\operatorname{div}(-\mathbf{k} \cdot \operatorname{grad} T)-\alpha \mathbf{D}: \mathbf{I}: \dot{\boldsymbol{\varepsilon}}$

\section{CORRIGE}

Eq. 2: $\rho C \dot{T}=-\operatorname{div}(-\mathbf{k} \cdot \operatorname{grad} T)-\alpha T_{0} \mathbf{D}: \mathbf{I}: \dot{\boldsymbol{\varepsilon}}$

The online version of the original article can be found under doi:10.1007/s11012-007-9105-3.

R. Ardito $(\varangle) \cdot$ C. Comi · A. Corigliano · A. Frangi

Dipartimento di Ingegneria Strutturale, Politecnico di

Milano, Milan, Italy

e-mail: raffaele.ardito@polimi.it

C. Comi

e-mail: claudia.comi@polimi.it

A. Corigliano

e-mail: alberto.corigliano@polimi.it

A. Frangi

e-mail: attilio.frangi@polimi.it 